\title{
Non-naturalized alien plants receive fewer flower visits than naturalized and native plants in a Swiss botanical garden
}

\author{
Mialy Razanajatovo $^{\mathrm{a}, *}$, Christine Föhr ${ }^{\mathrm{b}, \mathrm{c}}$, Markus Fischer ${ }^{\mathrm{b}, \mathrm{c}}$, Daniel Prati ${ }^{\mathrm{b}}$, Mark van Kleunen ${ }^{\mathrm{a}}$ \\ ${ }^{a}$ Ecology, Department of Biology, University of Konstanz, Universitätsstrasse 10, Konstanz D-78457, Germany \\ ${ }^{\mathrm{b}}$ Institute of Plant Sciences, University of Bern, Altenbergrain 21, Bern CH-3013, Switzerland \\ ${ }^{\mathrm{c}}$ Botanical Garden of the University of Bern, Altenbergrain 21, Bern CH-3013, Switzerland
}

\begin{abstract}
A B S T R A C T
Many animal pollinated plant species have been introduced to non native regions without their usual pollinators. Nevertheless, some of these alien species managed to establish reproducing naturalized pop ulations, which might negatively affect native plants. Recent studies have shown that many naturalized alien species can readily attract native pollinators. However, it is not known whether alien species that have not established naturalized populations are less successful in attracting pollinators. Therefore, we tested whether flower visitation rates are lower for non naturalized aliens than for naturalized alien and native species. We conducted a comparative study on flower visitation of 185 native, 37 naturalized alien and 224 non naturalized alien plant species in the Botanical Garden of Bern, Switzerland. Our phylogenetically corrected analyses showed that non naturalized alien species received fewer flower visitors than both naturalized alien and native species. Native, naturalized alien and non naturalized alien species were visited by similar flower visitor communities. Furthermore, among the naturalized alien species, the ones with a broader distribution range in Switzerland received a more diverse set of flower visitors. Although it has been suggested that most alien plants can readily integrate into native plant pollinator networks, we show evidence that the capacity to attract flower visitors in non native regions is different for naturalized and non naturalized alien plants. Therefore, we conclude that successful naturalization of alien plants may be related to flower visitation.
\end{abstract}

\section{Introduction}

Many plant species have been introduced, for example as gar den plants, to new regions where they encounter novel abiotic and biotic conditions. Some of these alien plant species managed to establish reproducing naturalized populations and some of those species have become invasive and constitute a threat to native communities by displacing native organisms and altering ecosystem functioning (Pimentel et al., 2000; Vilà et al., 2011; Vitousek et al., 1997). In order to predict and prevent invasions, and therefore to allow natural resource managers and policy makers to set priorities in management policies, it is of both basic and applied interest to understand the invasion process. Consequently, a major question in ecology and conservation biol ogy is what determines successful naturalization of alien species.

The establishment of alien species obviously depends, at least partly, on successful reproduction. Globally, an estimated $87.5 \%$

\footnotetext{
* Corresponding author. Tel.: +497531 884305.

E-mail address: mialy.razanajatovo@uni-konstanz.de (M. Razanajatovo).
}

of flowering plant species rely fully or partly on animals for pollination (Ollerton et al., 2011). Baker (1955) posed that the ability to attract pollinators in a new range is an important constraint for establishment, particularly if the species does not have the means of uniparental reproduction. Nevertheless, many alien plant species have managed to reproduce and establish natu ralized populations in new ranges without their usual pollinators, and some have become invasive. Recent studies showed that many naturalized and invasive alien plant species are capable of autono mous self fertilization (Harmon Threatt et al., 2009; van Kleunen et al., 2008), which makes them less reliant on pollinators. Other recent studies showed that many naturalized and invasive alien plant species have managed to integrate into native plant pollina tors webs, can use a range of different pollinators (Memmott and Waser, 2002; Vilà et al., 2009), and might negatively affect the pollination of co occurring native plants (Morales and Traveset, 2009). In other words, naturalized alien species are able to attract pollinators in their new ranges, and this might have allowed them to establish and maintain wild populations. However, none of these studies looked at flower visitation of alien species that have 
not become naturalized. Therefore, it remains unclear whether the capacity of attracting pollinators distinguishes naturalized alien from non naturalized alien species, that is, whether it could drive naturalization of alien species (van Kleunen et al., 2010).

The capacity of a plant to attract flower visitors is influenced by many factors, such as flower size and color, plant size, and area covered by and density of the plant population (Faegri and Van der Pijl, 1966; Schemske and Bradshaw, 1999; van Kleunen et al., 2007b). Many alien plants have showy and attractive flowers due to a biased introduction of such species (van Kleunen et al., 2007a). This means that species traits that are associated with naturalization and invasiveness might actually be related to human preference and selection for introduction on ornamental or cultivation purposes (Chrobock et al., 2011). Thus, it is impor tant to account for these factors when comparing flower visitation among groups of species.

To test the importance of flower visitation for naturalization of alien plant species, we used a powerful multi species comparative approach (van Kleunen et al., 2014) by assessing visitation rates on 446 alien and native plant species in the Botanical Garden of Bern, Switzerland. Botanical gardens offer unique opportunities for host ing comparative studies because species from a broad taxonomic range and from a wide geographic area are growing under similar and thus comparable conditions (Primack and Miller Rushing, 2009). Flower visitation might differ between garden habitats and (semi )natural habitats (Chrobock et al., 2013b). However, as many naturalized alien species often escaped from botanical gar dens (Hulme, 2011), it is highly relevant to study the potential drivers of naturalization of alien species in such garden habitats.

Species that are alien to Switzerland but native to other parts of Europe might be more likely to encounter suitable flower visitors as it is likely that many of the flower visitors in their native range also occur in Switzerland. Therefore, we also tested whether flower visitation differed between alien species of European origin and the ones of non European origins. It has been shown that flower visita tion may change along the different stages of the invasion process as a newly introduced species may receive fewer visits at an early stage (King and Sargent, 2012). Therefore, we also tested among our naturalized alien species, whether the ones that occur more frequently in Switzerland attract more flower visitors. Because effective pollen transfer is most likely determined by the number of insect visits, the duration of each visit and the diversity of flower visitors (Herrera, 1989; Ollerton et al., 2007; Vázquez et al., 2005), we assessed these different parameters.

Our specific questions were: (1) Is flower visitation (number of visits, duration of visits, flower visitor diversity) lower for non nat uralized species than for naturalized alien and native species? (2) Are native, naturalized alien and non naturalized alien plant species visited by different insect communities? (3) Do alien species from other parts of Europe attract more flower visitors than alien species from other continents? (4) Is flower visitation higher for more wide spread than for less widespread naturalized aliens in Switzerland?

\section{Methods}

\subsection{Study site and flower visitor observations}

To test whether native, naturalized alien and non naturalized alien plant species differ in number of insect visits, duration of vis its, diversity of flower visitors and composition of flower visitor community, we conducted observations of flower visitors to 185 native, 37 naturalized alien, and 224 non naturalized alien plant species (Table S1) in the Botanical Garden of Bern, Switzerland $\left(46.57^{\circ} \mathrm{N}, 7.26^{\circ} \mathrm{E}\right)$. The botanical garden contains about 4500 plant species growing outdoors, mostly in mixed garden beds, in a total area of $24470 \mathrm{~m}^{2}$ under similar climatic conditions (altitude:
$501537 \mathrm{~m}$, rainfall: $1028 \mathrm{~mm} /$ year, annual mean temperature: $8.1^{\circ} \mathrm{C}$ ). The garden is situated near the city center of Bern, along the River Aare, which provides a green corridor connecting it to surrounding (semi )natural habitats. We recorded all native, naturalized alien and non naturalized alien entomophilous plant species that were flowering in the botanical garden during the observation periods, and from each of these three groups we randomly selected species for observations.

To cover the complete plant flowering season, we did seven observation censuses: 24 March, 19 April, 24 May, 27 June, 29 July, 29 August and 23 September 2011. At each census, we did observations on natives, naturalized and non naturalized aliens (Table S2), and each species was used in one census only (Table S1). A few days prior to each observation census, we prepared a list of the flowering entomophilous species in the botanical garden at that time. To account for potential confounding factors that could influence flower visitation, we measured species' characteristics that are likely to determine how conspicuous and attractive they are to flower visitors. For each species, we recorded maximum plant height, total area occupied in the botanical garden (i.e. abundance of the species in the garden), the number of flower units per $\mathrm{m}^{2}$ (i.e. flower unit density), size of flower units, flower color categories (blue, green, red, white, yellow), flower symmetry (bilaterally or radially symmetric) and exposure to the sun at the moment of observation (yes/no). A flower unit was defined as a unit of one or more flowers that an insect has to fly to in order to reach the next unit (Dicks et al., 2002). So, one capitulum with multiple flowers of an Asteraceae species was considered to be one flower unit. Our flower color categories might not capture all the optical cues that are relevant for pollinators. We documented the origin and the status of each species, i.e. whether it is native to Europe or not (Tutin et al., 1980), and whether it is a native, naturalized alien or non naturalized alien species in Switzerland (Lauber and Wagner, 2007).

Because flower visitor activity depends strongly on the weather, each census was carried out on a sunny day. Furthermore, to reduce variation in flower visitation due to diurnal changes in weather conditions, we did all observations within the short time frame of approximately two hours. We chose the time frame c. $13001500 \mathrm{~h}$ because most flower visitors in the botanical garden were then active. Therefore, for each census, a team of 715 volun teers (a total of 34 persons for the whole study), which consisted of students, lab members and colleagues from other research labs, did the observations simultaneously. For each flowering species, ten flower units, if available, were observed simultaneously for $15 \mathrm{~min}$. All flower units were in close proximity but were not nec essarily on the same individual. If there were fewer than 10 flower units available, we recorded the number of observed flower units.

All flower visitors that made contact with reproductive organs of the focal flower units were assumed to be pollinators. Since the observations were performed by volunteers without specific taxonomic knowledge on insects, and we could not catch all flower visitors, it was not possible to identify flower visitors to the species level. We recorded the number of visits, the duration of visits, using a watch, and the flower visitor taxonomic groups (ants, bee flies, bees, beetles, bumblebees, butterflies, flies, hover flies, moths and wasps). We recorded duration of visits because long vis itation periods may increase the likelihood of the flower visitor to function as pollinators (Ollerton et al., 2007). In total, we observed flower visitors for $111.5 \mathrm{~h}$. Although $15 \mathrm{~min}$ of observation per species will not have given a complete picture of flower visitation to each of the individual species, the objective of our study was to compare the groups of native, naturalized alien and non naturalized rather than the individual species. Therefore, we chose to maximize the number of species (i.e. the most relevant unit of replication) over the time per species as this increases the 
statistical power for the comparison among the different plant groups (see simulations by van Kleunen et al. (2014)).

\subsection{Phylogeny}

Our set of 446 study species covered 64 plant families. Therefore, they share different degrees of evolutionary history; e.g. confamilial or congeneric plants might have more similar flower visitation rates than less related species. To account for this phylogenetic non independence of our species in the analyses (Felsenstein, 1985), we constructed a phylogeny of the species using the most recent megatree available in the program Phylomatic online version 3 (Webb and Donoghue, 2005). We further resolved polytomies within the Phylomatic phylogenetic tree using published phylogenies based on DNA studies (Table S3). We finally used the tree with the best resolution possible. We estimated the branch length within our resulting tree using Phylocom (Wikström et al., 2001).

\subsection{Data analysis}

\subsubsection{Visitation of native, naturalized and non naturalized plants}

To compare the number of visits, the duration of visits and the number of flower visitor groups during the 15 min observation period among non naturalized alien, naturalized alien and native species, we fitted linear mixed models (Laird and Ware, 1982) using the lme function of the nlme package (Pinheiro et al., 2013) in the $\mathbf{R}$ software, version 2.15.2 (R Core Team, 2012). To improve normality, we $\log$ transformed the response variables. We transformed and analyzed our count data using Gaussian error distributions rather than using Poisson distributions in order to be able to account for phylogenetic non independence among species. As we included 185 native, 37 naturalized alien and 224 non naturalized alien plant species, the total sample size was 446 . As an additional measure of the diversity of flower visitors, we also calculated the Shannon diversity index based on flower visitor groups, but this was only possible for the plant species that received visits (139 native, 32 nat uralized alien and 160 non naturalized alien; i.e. a total sample size of 331). This measure of diversity accounts for the fact that a plant visited by a bee species and a butterfly species has a more diverse visitor assemblage than a plant visited by two different bee species, and thus provides an estimate of the functional diversity of the pollinators (Ollerton et al., 2007). To account for the fact that some species had fewer than 10 observed flowers, we fitted the number of observed flower units as a fixed term before plant species status (Jasieński and Bazzaz, 1999).

To correct for phylogenetic non independence of species, for the analyses of the number of visits, the duration of visits, and the number of flower visitor groups during the 15 min observation period, we additionally fitted models including the phylogenetic correlation structures corPagel (Freckleton et al., 2002) and corGra fen (Grafen, 1989) in the ape package (Paradis et al., 2004). When we included phylogeny in the analysis of the Shannon indices, models did not converge. Therefore, we used for the analysis of that variable the lmer function in the lme4 package in $\mathrm{R}$ (Bates and Maechler, 2009), and accounted for taxonomy instead of phy logeny by including plant family as an additional random factor.

As alien species have frequently been introduced for ornamental purposes, many traits that might affect attractiveness to flower visitors may differ between alien and native species. Indeed, naturalized alien and native species had a significantly higher number of flower units per $\mathrm{m}^{2}$ than the non naturalized aliens. Non naturalized aliens also had significantly smaller flower units than native and naturalized alien species (Table S4). To correct for these differences among plant groups, and to test how these traits affect flower visitation, we additionally included log plant height, log total area covered by the species, log number of flower units per $\mathrm{m}^{2}, \log$ diameter of a flower unit, exposure to the sun, symmetry of the flower and flower color category before species status (native, naturalized, non naturalized) as fixed terms. We log transformed many of the covariates to linearize the relationships between the response variables and these covariates. Furthermore, to reduce col linearity among explanatory variables and to facilitate comparisons among estimates, the covariates were centered and scaled to a mean of zero and a standard deviation of one (Schielzeth, 2010). To be able to average our results across the different flower color categories, we replaced the multinominal variable flower color category by four dummy variables (Schielzeth, 2010). These dummy variables were blueness (i.e. flower was blue or was not blue), greenness, redness and whiteness. To account for heteroscedasticity, we added a fixed variance structure in which the variance was proportional to log plant height for number of insect visits and number of flower visitor groups, and proportional to number of observed flower units for duration of visits (Zuur et al., 2009). We used model selection based on the Akaike Information Criterion AIC (Zuur et al., 2009) to decide which covariates to keep in the final model. We always kept the main factor of interest, species status, in the final models. Signifi cances of the fixed terms in the final models were tested using log likelihood ratio tests (Zuur et al., 2009). As we did the observa tions on seven census dates, we accounted for this in all models by including the census date as a random term.

\subsubsection{Visitation of alien plants from European and non European origins}

To test whether alien species that are native to other parts of Europe attract more flower visitors than alien species from other continents, and whether successful naturalization of alien species depends on their European origin or not, we also fitted linear mixed models using the subset of alien species $(n=261)$. In these models, we used the same covariates as in the previous models and instead of species status, as main factors of interest, we included European origin, naturalization status of the alien species (yes/no), and the interaction term European origin $\times$ naturalization status.

\subsubsection{Visitation of naturalized alien plants differing in frequency of occurrence in Switzerland}

To test whether the naturalized alien plant species that occur more frequently in Switzerland show higher flower visitation (number of visits, duration of visits, flower visitor diversity), we analyzed the subset of naturalized aliens $(n=35)$. We fitted linear mixed models using the lme function in R. In these models, we used the same covariates as in the previous models, and as main factor of interest, we included the log number of grid cells $(5 \mathrm{~km} \times 5 \mathrm{~km})$ in which the species has been recorded since 1994 in Switzerland (http://www.infoflora.ch/de/flora/art abfragen. html) instead of species status. This is also representative for other parts of Central Europe, as the number of occupied grid cells of the naturalized aliens in Switzerland is positively correlated with the number of grid cells (quarters of c. $12 \mathrm{~km} \times 11 \mathrm{~km}$ ) occupied by these species in Germany (Kendall's tau $=0.439, p=0.0008$; data from http://www.floraweb.de/pflanzenarten/pflanzenarten. html).

\subsubsection{Flower visitor communities on native, naturalized and non naturalized plants}

To test whether non naturalized alien, naturalized alien and native plant species are visited by different insect communities, we fitted a multinomial logistic regression (Ntzoufras, 2011) in WinBUGS (Lunn et al., 2000). Our multinomial model included the number of each flower visitor group (ants, bee flies, bees, beetles, bumblebees, butterflies, flies, hover flies, moths, and 
wasps) as a multi categorical response variable. As this model was difficult to fit, we did not use all the covariates of the previous models but we only included plant species status as a fixed term, and date of observation as a random term. Because we wanted to look at the composition of the flower visitor communities, we considered only plant species that received visits $(n=339)$. We additionally included log plant height as a fixed term in the model because it influenced significantly the number of visits, the number of flower visitor groups and the Shannon indices of flower visitor groups. To account for taxonomy, we added plant family as a random factor in the model. Furthermore, for each flower visitor separately, we analyzed the average duration per individual visit by fitting linear mixed models. This was only possible for six flower visitor groups (ants, bees, beetles, bumblebees, flies, hoverflies and wasps) which visited the plants in sufficient numbers.

\section{Results}

3.1. Number of insect visits to native, naturalized alien and non naturalized alien plant species

Non naturalized alien species received on average significantly fewer insect visits per flower than naturalized alien species, and the latter received as many visits as native species (Table S5). This pattern remained the same after correction for phylogenetic non independence of species and plant and flower characteristics (Table 1, Fig. 1a). The number of insect visits increased significantly with number of observed flower units, plant height and flower unit diameter, and was higher for flowers in the sun and for blue flowers (Table 1).

For the subset of alien species, the average numbers of insect visits per flower on European and non European species did not differ (Tables S6 and S7). For the subset of naturalized alien species, the number of insect visits was not significantly correlated with the frequency of occurrence in Switzerland (Table S8).

\subsection{Duration of insect visits on native, naturalized alien and non naturalized alien plant species}

Overall, the average duration of individual visits did not significantly depend on species status (Table S5). This remained the same after correction for phylogenetic non independence of species and plant and flower characteristics (Table 1, Fig. 1b).
The average duration of individual visits increased with the num ber of observed flower units and flower unit diameter, and was shorter for plants with white flowers than for plants with flowers of other color categories (Table 1 ).

For the subset of alien species, duration of visits did also not differ between European and non European species (Tables S6 and S7). The average duration of individual visits among the naturalized alien species was slightly positively correlated with the number of occupied grid cells in Switzerland, but this was statistically not significant ( $p=0.0539$, Table S8).

\subsection{Flower visitor diversity of native, naturalized alien and non naturalized alien plant species}

There was no significant difference in the number of flower visitor groups among native, naturalized alien and non naturalized alien species. There was also no significant difference in the Shannon indices of flower visitor groups among native, naturalized alien and non naturalized alien species (Table S9). After correction for phylogenetic non independence of species and plant and flower characteristics, we found that non naturalized alien species tended to receive a less diverse set of flower visitors, both in terms of the number and the Shannon index of flower visitor groups, than naturalized alien and native species but this was not statistically significant $(p<0.1$, Tables 1 and 2, Fig $1 \mathrm{c}$ and d). The number of flower visitor groups increased significantly with number of observed flower units, plant height and flower unit diameter, and was higher for flowers in the sun (Table 1). Similarly, the Shannon diversity index of flower visitor groups increased with plant height and number of flower units per $\mathrm{m}^{2}$, and was significantly higher for blue flowers (Table 2).

Among the naturalized aliens, there was no significant differ ence in the number of flower visitor groups between European species and species from non European origin. There was also no significant difference in the Shannon indices of flower visitor groups between European species and species from non European origin (Table S10). After correction for phylogenetic non indepen dence of species and plant and flower characteristics, we found that European species tended to receive a more diverse set of flower visitor groups but this was not statistically significant ( $p=0.0718$ for the Shannon indices, Table S11). Furthermore, naturalized alien species with a broader distribution range in Switzerland received a more diverse set of flower visitors than

Table 1

Results of three phylogenetically corrected linear mixed-effects models testing how the number of insect visits, the average duration of individual visits, and the number of flower-visitor groups depend on traits related to conspicuousness of the plants and flowers and on species status (native, naturalized alien, non-naturalized alien). Final models from model selections based on the Akaike Information Criterion are presented.

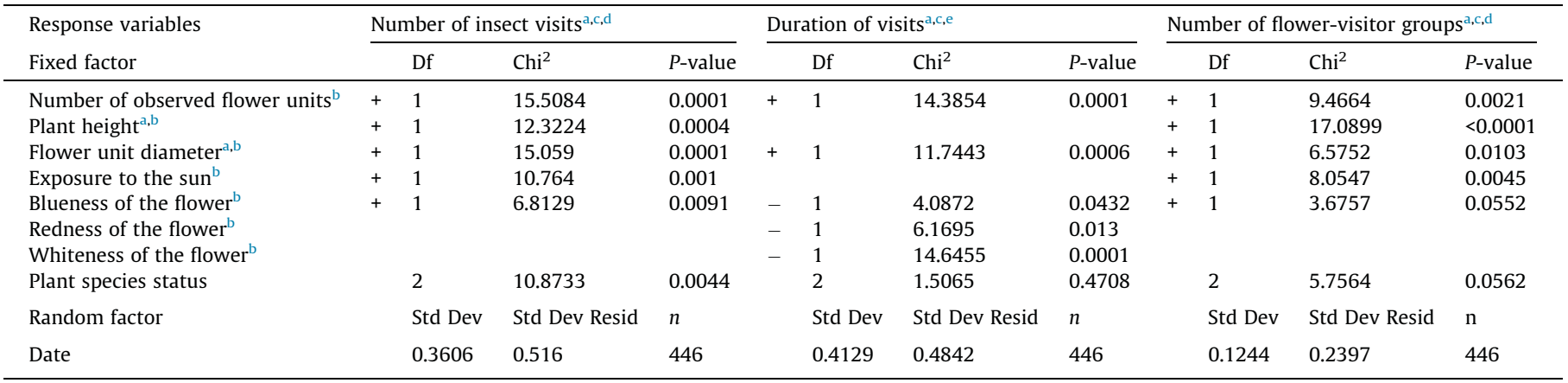

+ - Indicates the directionality of the effect.

a Variables were log transformed.

b Variables were centered and scaled.

c Phylogenetic Grafen's correlation structure (respectively, rho $=0.0227,0.0239,0.0143$ ).

d Variance structure: fixed weights plant height.

e fixed weights number of observed flower units. 

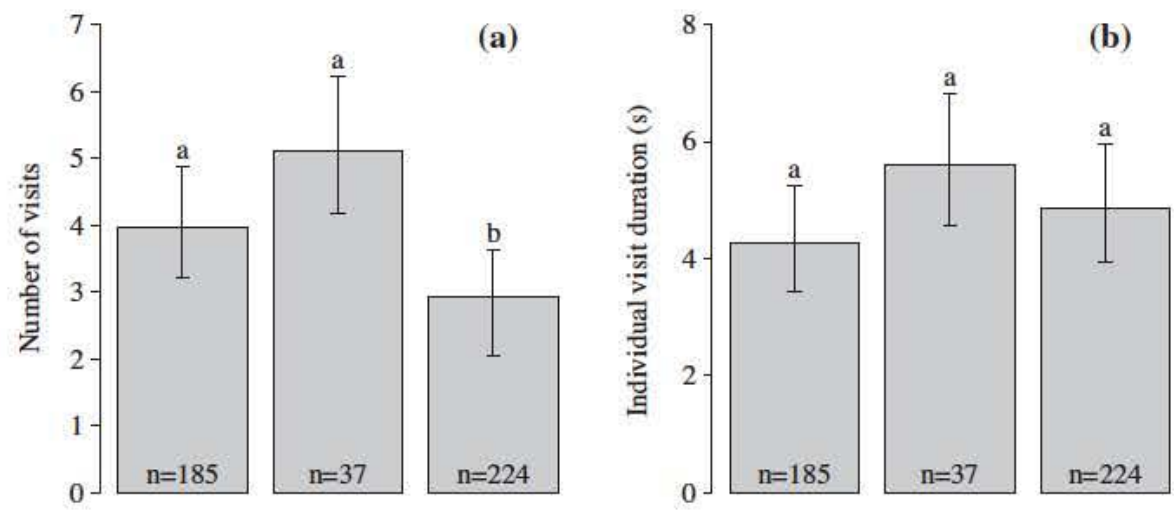

(c)
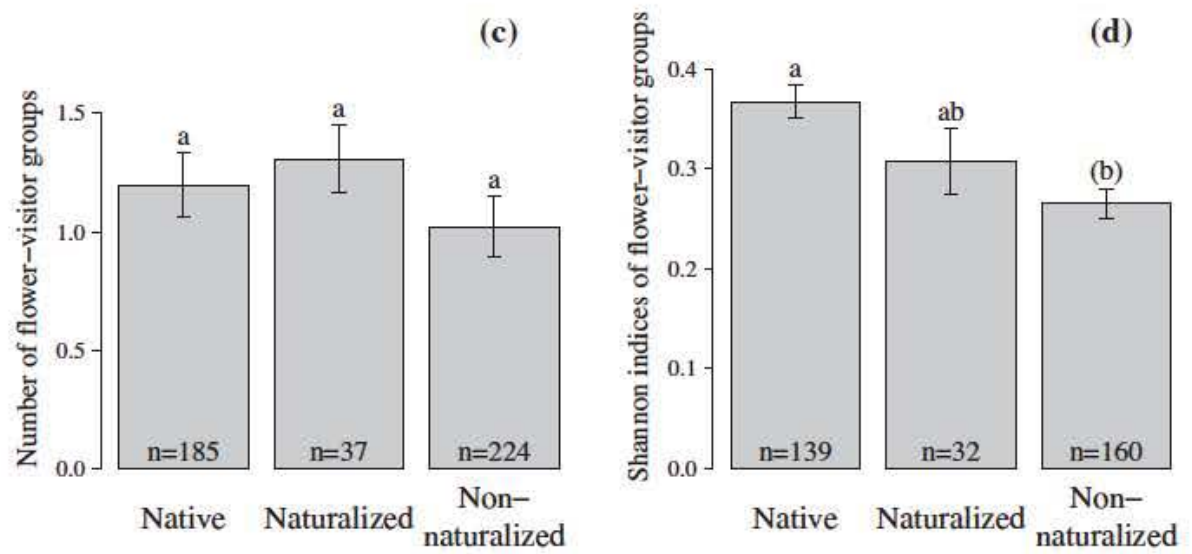

Fig. 1. (a) Total number of insect visits, (b) average duration of individual visits, (c) number of flower-visitor groups, and (d) Shannon-diversity indices of flower-visitor groups to native, naturalized alien and non-naturalized alien plant species in 15 min. Estimated means $+1-$ SE from phylogenetically corrected linear mixed models. Differences significant at $p<0.05$ are indicated by different letters above the bars, $p<0.1$ is indicated by the presence of brackets around the letter.

Table 2

Results of a linear mixed model testing how the Shannon-diversity index of flowervisitor groups depends on traits related to conspicuousness of the plants and flowers, and on status (native, naturalized and non-naturalized alien). A final model from a model selection based on the Akaike Information Criterion is presented.

\begin{tabular}{|c|c|c|c|c|}
\hline Fixed factor & & Df & $\mathrm{Chi}^{2}$ & $P$-value \\
\hline Plant height $t^{a, b}$ & + & 1 & 13.103 & 0.0003 \\
\hline Area covered by the species ${ }^{\tilde{a}, b}$ & + & 1 & 2.0852 & 0.1487 \\
\hline Number of flower units per $m 2^{a, b}$ & + & 1 & 7.8624 & 0.005 \\
\hline Flower unit diameter & + & 1 & 3.2963 & 0.0694 \\
\hline Exposure to the sun ${ }^{\mathrm{b}}$ & - & 1 & 2.0242 & 0.1548 \\
\hline Blueness of the flower ${ }^{b}$ & + & 1 & 3.8429 & 0.05 \\
\hline Whiteness of the flower ${ }^{b}$ & + & 1 & 3.8199 & 0.0507 \\
\hline Plant species status & & 2 & 5.683 & 0.0583 \\
\hline Random factor & & Std Dev & Std Dev Resid & $n$ \\
\hline Date & & 0.0223 & 0.3609 & 331 \\
\hline Family & & $<0.0001$ & 0.3609 & 331 \\
\hline
\end{tabular}

+1 - Indicates the directionality of the effect.

a Variables were log transformed.

b Variables were centered and scaled.

the ones with a smaller range, as indicated by a significant effect on the Shannon diversity index $(p=0.0003$, Table S12).

\subsection{Flower visitor communities}

We observed ten different groups of flower visitors on the plants, and bees were the most frequent visitors of our plant species (Fig. 2). Overall, native, naturalized alien and non naturalized alien plants were visited by similar communities of flower visitor groups. The three categories of plant species were, to some extent, likely to be visited by all the observed flower visitor groups, and the probabilities of visits to native, naturalized and non naturalized

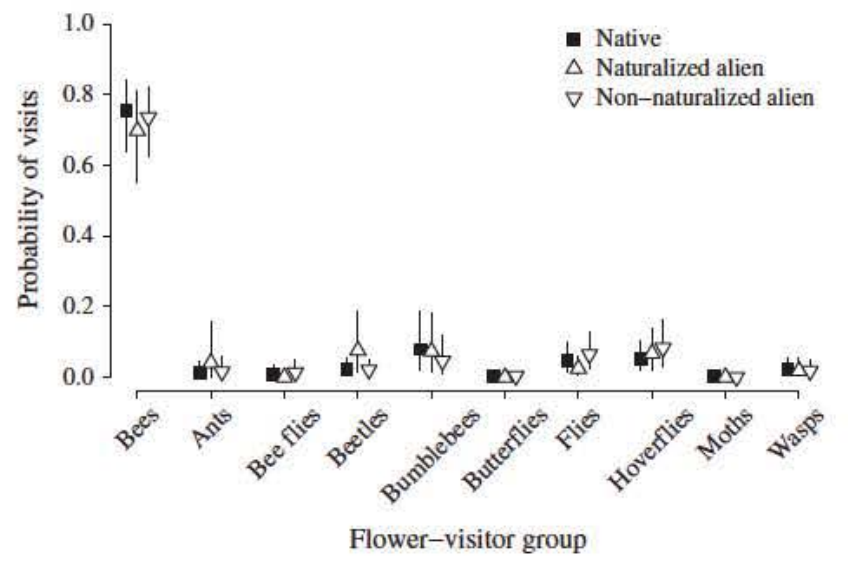

Fig. 2. Probability of visits by different flower-visitor groups to native, to naturalized alien and to non-naturalized alien plant species. Bars indicate 95\% credible intervals of a multinomial logistic regression.

plants did not significantly differ within each flower visitor group (Fig. 2). There was also no significant difference in the duration of visit by each flower visitor group among the three categories of plant species, except for the ants, as naturalized aliens were visited for shorter time by ants compared to non naturalized aliens and natives (Table S13).

\section{Discussion}

Based on observations on invasive alien plant species, it has been suggested that most alien plants can readily integrate into native plant pollinator networks (Richardson et al., 2000). However, in 
our botanical garden study, we found that non naturalized alien plants attracted significantly fewer flower visitors than both natu ralized alien and native plants. Furthermore, among the naturalized alien plants, the ones that attracted a more diverse set of flower visitors were also more widespread in Switzerland. Although one cannot generalize from the observations at one location, our results suggest that the ability to attract flower visitors plays an important role during the invasion process of alien plants.

\subsection{Flower visitation is lower for non naturalized than for naturalized alien and native plants}

Our results show that non naturalized aliens received fewer flower visits than naturalized aliens. In another recent study, on a total of 17 plant species, there was no difference in flower visita tion to confamilial naturalized and non naturalized alien species, but both received fewer visits than confamilial natives (Chrobock et al., 2013b). In our study on a much larger number of species, naturalized alien species received, on average, at least as many vis its as native species. Possibly, the naturalized species used by Chrobock et al. (2013b) received fewer visits than the native species, because not all naturalized species were naturalized at the local scale in the actual study region. Whatever the reason for the discrepancy, we here provide the first evidence that the capacity to attract flower visitors in a non native region can be different for naturalized and non naturalized alien plants.

\subsection{Native, naturalized and non naturalized alien plants are visited by similar flower visitor communities}

Our results suggest that flower visitor groups were shared among native, naturalized alien and non naturalized alien plant species, and the major flower visitor group were bees (Fig. 2). The set of study species were likely to receive visits of the same available flower visitor groups in our study site, which suggests that differences in the number of visits are not due to differences in the identity of the flower visitor types. Some flower visitor groups may be less efficient pollinators than others, depending on their morphology and on the floral morphology (Ollerton et al., 2007). Although we did not identify the flower visitors at the species level, most bees the most frequent flower visitor group of our three categories of plant species were honeybees, Apis mellifera (personal observation). Therefore, our findings are in line with the idea that generalist pollinators such as A. mellifera play an important role in the pollination of alien plants (Richardson et al., 2000). Nevertheless, pollinator communities might vary among locations. Future studies should thus compare flower visitation among native, naturalized alien and non natural ized alien species in other botanical gardens or in the wider countryside using a common garden approach and being cautious not to cause undesired new establishment.

\subsection{Alien species from other parts of Europe do not attract more flower} visitors than alien species from other continents

Because insect communities in Switzerland are likely to be more similar to those in other European countries than to those on other continents, we expected that alien plant species native to other European countries would attract more flower visitors than alien species that are not native to Europe. In a recent study, on 119 plant species, some flower visitors preferred Palaearctic plants whereas others preferred the non Palaearctic ones in urban gardens in England (Hanley et al., 2014). However, that study was restricted to observations of bumblebee species only. Moreover, the study did not distinguish between naturalized plant species and the ones that have not managed to establish reproducing populations in the wild. In our study, on a total of 261 naturalized and non naturalized alien plant species, we found little support for the hypothesis that species from Europe receive more flower visits than species from other continents. Therefore, we conclude that the origin of the alien plant species is of limited importance for the attraction of flower visitors, at least in our study system.

\subsection{Flower visitation is higher for more widespread naturalized alien species in Switzerland}

It is likely that alien plants became naturalized because they manage to attract suitable flower visitors. Therefore, we expected that naturalized aliens that occur more frequently in Switzerland would show higher flower visitation. Indeed, we found that natural ized aliens that are more widespread in Switzerland attracted a more diverse set of flower visitors than the less widespread ones $(p=0.0003$, Table S12). Attracting a more diverse set of flower visi tors is likely to increase seed set because the likelihood that one of the flower visitors functions as an effective pollinator increases and because of the complementarity of the functional groups of flower visitors (Ollerton et al., 2007). Moreover, plant species that can attract a wider variety of flower visitors are also more likely to encounter one or more of these visitors outside the botanical garden.

It has been suggested that most naturalized alien plants are generalized with regard to pollinators because this will facilitate pollinator attraction in the introduced range (Richardson et al., 2000). A few specialized alien plants have also become invasive, but only in regions where suitable pollinators were also introduced (Kaiser Bunbury et al., 2011; Morales and Aizen, 2006) or naturally present (Rodger et al., 2010, 2013), or when the plant can also self fertilize (Ollerton et al., 2012). A recent study showed that among 118 native European grassland species, there was no relationship between their generalization towards pollinators and invasiveness in other parts of the world (Chrobock et al., 2013a). So, although having a specialized pollination system does not necessarily ham per invasion, our results suggest that naturalized species and particularly more widespread ones are more generalized than non naturalized species in their introduced range.

\subsection{What drives differences in flower visitation?}

Flower visitation is related to many factors such as environmen tal conditions and plant traits (Faegri and Van der Pijl, 1966). For example, the activity of pollinators depends on meteorological parameters such as temperature, light intensity, humidity and wind speed (Kevan and Baker, 1983). Indeed, in our study, flowers in the sun received more visits than those in the shade. Conspicu ousness of plants is also likely to affect flower visitation. Plant height was positively correlated to the number of flower visitors, number and diversity of flower visitor groups, and size of the floral units was positively correlated to the number of flower visitors and visitation duration. In our study, blue flowers were more attractive to flower visitors than flowers with other colors. This color effect may be driven by bees, which were the most frequent visitors in our study, and which frequently prefer the blue wavelengths (Hsu and Yang, 2012). Our study thus confirms that environmental factors and species traits affect flower visitation rates.

In our analyses, with as well as without corrections for all the traits that significantly affected flower visitation, we found similar results for the comparisons of flower visitation rates among native, naturalized alien and non naturalized alien plant species. The results were also relatively robust with regard to phylogenetic corrections, which is in line with the weak phylogenetic signals in our results (see Grafen's rho values below Table 1). This indicates that differences in visitation among native, naturalized alien and non naturalized alien plants may be due to traits or 
combinations thereof that we could not account for in our analy ses. Possibly, many of the non naturalized alien species in our study may not have the quantity and quality of rewards that are essential for attracting flower visitors in central Europe, while the naturalized species happen to have such rewards. For example, the invasive alien plant Impatiens glandulifera is known to monopolize native pollinators in its introduced range because of its extremely sugar rich nectar (Chittka and Schurkens, 2001). Unfortunately, we could not assess nectar production and compo sition, because it was too difficult to extract nectar from most of the study species, and we were not allowed to destructively sample flowers in the botanical garden. However, if the production of floral rewards is phylogenetically conserved (Ornelas et al., 2007), the inclusion of a phylogenetic correction in our analyses should have largely accounted for variation in floral rewards among the study species. Nevertheless, future studies should also explicitly consider the role of floral rewards in the naturalization success of plants.

\subsection{Non naturalized alien plants may be pollinator limited}

Our results suggest that alien plant species that have not estab lished self sustaining populations may be pollinator limited because they attracted fewer flower visitors than naturalized alien and native species. However, flower visitation does not necessarily increase seed production, as not all flower visitors are effective poll inators. Moreover, as the relationship between seed set and flower visitation is most likely asymptotic, the lower visitation of non nat uralized plants might have been in the asymptotic region, and thus may not limit seed production. Furthermore, pollen limitation is not only driven by flower visitation rates but also by mate availability. For many of our study species, it was difficult to control the avail ability of mates in the botanical garden. Therefore, we could not do a pollen supplementation experiment to test for pollen limitation of seed production. Future studies should thus directly test whether seed production is more pollinator limited in non naturalized alien than in naturalized and native species. Furthermore, it cannot be excluded that flower visitation is correlated with other factors that affect naturalization success. For example, most naturalized species might have received more propagule pressure than the non natu ralized ones if the latter have only been introduced in the botanical garden. Therefore, future studies are required that test for a direct causal relationship between flower visitation and establishment success of alien plants. Nevertheless, our study on a large number of species suggests that non naturalized alien plants, in contrast to naturalized and native ones, may be pollinator limited.

\subsection{Alien plants may impact native plants and pollinators}

Our findings support others that some alien plants can readily attract flower visitors in the non native range (Memmott and Waser, 2002). This might suggest that naturalized alien plants play an important role in sustaining native pollinator communi ties. It has been shown that alien plants have a predominantly negative effect on flower visitation of co occurring native plants. Indeed, flower visitation is frequently reduced on the natives due to higher attractiveness of the aliens (Morales and Traveset, 2009). This is obviously detrimental to the natives because it may decrease their reproductive success (Brown et al., 2002; Morales and Traveset, 2009). On the other hand, the presence of an alien plant can in certain situations also increase flower visita tion of co occurring natives. Indeed, the aliens can act as magnet species, attracting more pollinators that spill over to natives that are in the vicinity (Lopezaraiza Mikel et al., 2007). Likewise, the presence of an alien plant can also have a facilitative effect on flower visitation of other co occurring aliens, and therefore might promote their naturalization (Molina Montenegro et al., 2008). Nevertheless, increased flower visitation does not necessarily increase reproductive success, as there are increasing chances for heterospecific pollen transfer (Lopezaraiza Mikel et al., 2007).

\subsection{Conclusions}

Our study shows that entomophilous alien plants that have not established naturalized populations are less successful than naturalized alien and native plants in attracting flower visitors. This may suggest that successful naturalization is related to flower visitation. An alternative interpretation could be that naturaliza tion success is driving visitation, rather than the other way around. This could happen if flower visiting insects are already more used to naturalized than to non naturalized plants in the landscape. However, given that the Botanical Garden of Bern is already over 150 years old, we assume that the local pollinator community has had enough time to get used to both the naturalized and non naturalized alien species in the garden. This is among the reasons why the botanical garden is certainly a unique habitat to study flower visitation of naturalized and non naturalized plants. Our study on flower visitation of 446 species strongly suggests that non naturalized alien plants, in contrast to naturalized and native ones, might be pollinator limited.

\section{Acknowledgements}

We thank all volunteers for the data collection, the Plant Ecol ogy group at the University of Bern for hosting MR, the Botanical Garden of Bern for allowing the pollinator observations, Anne Kempel, Thomas Chrobock, Wayne Dawson and Rudolf Rohr for Statistics advice, the Swiss Federal Commission for Scholarships for Foreign Students, and the International Max Planck Research School for Organismal Biology for supporting MR, the Deutsche Forschungsgemeinschaft (Project KL1866/3 1) for funding; Noëlie Maurel and two anonymous reviewers for helpful comments on a previous version of the manuscript.

\section{References}

Baker, H.G., 1955. Self-compatibility and establishment after long distance dispersal. Evolution 9, 347-349.

Bates, D., Maechler, M., 2009. lme4: linear mixed-effects models using S4 classes. R package version 0.999375-32.

Brown, B.J., Mitchell, R.J., Graham, S.A., 2002. Competition for pollination between an invasive species (purple loosestrife) and a native congener. Ecology 83, 2328-2336.

Chittka, L., Schurkens, S., 2001. Successful invasion of a floral market - an exotic Asian plant has moved in on Europe's river-banks by bribing pollinators. Nature $411,653$.

Chrobock, T., Kempel, A., Fischer, M., van Kleunen, M., 2011. Introduction bias: cultivated alien plant species germinate faster and more abundantly than native species in Switzerland. Basic Appl. Ecol. 12, 244-250.

Chrobock, T. Weiner, C.N., Werner, M., Bluethgen, N., Fischer, M., van Kleunen, M., 2013a. Effects of native pollinator specialization, self-compatibility and flowering duration of European plant species on their invasiveness elsewhere. J. Ecol. 101, 916-923.

Chrobock, T., Winiger, P., Fischer, M., van Kleunen, M., 2013b. The cobblers stick to their lasts: pollinators prefer native over alien plant species in a multi-species experiment. Biol. Invasions 15, 2577-2588.

Dicks, L., Corbet, S., Pywell, R., 2002. Compartmentalization in plant-insect flower visitor webs. J. Anim. Ecol. 71, 32-43.

Faegri, K., Van der Pijl, L., 1966. The Principles of Pollination Ecology. Pergamon Press, Oxford.

Felsenstein, J., 1985. Phylogenies and the comparative method. Am. Nat. 125, 1-15. 
Freckleton, R., Harvey, P., Pagel, M., 2002. Phylogenetic analysis and comparative data: a test and review of evidence. Am. Nat. 160, 712-726.

Grafen, A., 1989. The phylogenetic regression. Philos. Trans. Roy. Soc. Lond. Ser. B: Biol. Sci. 326, 119-157.

Hanley, M.E., Awbi, A.J., Franco, M., 2014. Going native? Flower use by bumblebees in English urban gardens. Ann. Bot. 113, 799-806.

Harmon-Threatt, A.N., Burns, J.H., Shemyakina, L.A., Knight, T.M., 2009. Breeding system and pollination ecology of introduced plants compared to their native relatives. Am. J. Bot. 96, 1544-1550.

Herrera, C.M., 1989. Pollinator abundance, morphology, and flower visitation rate: analysis of the "quantity" component in a plant-pollinator system. Oecologia 80, 241-248.

Hsu, P.-S., Yang, E.-C., 2012. The critical cue in pattern discrimination for the honey bee: color or form? J. Insect Physiol. 58, 934-940.

Hulme, P.E., 2011. Addressing the threat to biodiversity from botanic gardens. Trends Ecol. Evol. 26, 168-174.

Jasieński, M., Bazzaz, F.A., 1999. The fallacy of ratios and the testability of models in biology. Oikos 84, 321-326.

Kaiser-Bunbury, C.N., Valentin, T., Mougal, J., Matatiken, D., Ghazoul, J., 2011. The tolerance of island plant-pollinator networks to alien plants. J. Ecol. 99, 202213.

Kevan, P., Baker, H., 1983. Insects as flower visitors and pollinators. Annu. Rev. Entomol. 28, 407-453.

King, V.M., Sargent, R.D., 2012. Presence of an invasive plant species alters pollinator visitation to a native. Biol. Invasions 14, 1809-1818.

Laird, N.M., Ware, J.H., 1982. Random-effects models for longitudinal data Biometrics 38, 963-974.

Lauber, K., Wagner, G., 2007. Flora Helvetica, 3e éd. Traduction de l'allemand et adaptation E. Gfeller, Haupt, Berne-Stuttgart-Vienne.

Lopezaraiza-Mikel, M.E., Hayes, R.B., Whalley, M.R., Memmott, J., 2007. The impact of an alien plant on a native plant-pollinator network: an experimental approach. Ecol. Lett. 10, 539-550.

Lunn, D.J., Thomas, A., Best, N., Spiegelhalter, D., 2000. WinBUGS-a Bayesian modelling framework: concepts, structure, and extensibility. Stat. Comput. 10, $325-337$.

Memmott, J., Waser, N.M., 2002. Integration of alien plants into a native flowerpollinator visitation web. Proc. Roy. Soc. Lond. Ser. B: Biol. Sci. 269, 2395-2399.

Molina-Montenegro, M.A., Badano, E.I., Cavieres, L.A., 2008. Positive interactions among plant species for pollinator service: assessing the 'magnet species' concept with invasive species. Oikos 117, 1833-1839.

Morales, C.L., Aizen, M.A., 2006. Invasive mutualisms and the structure of plantpollinator interactions in the temperate forests of north-west Patagonia Argentina. J. Ecol. 94, 171-180.

Morales, C.L., Traveset, A.,2009. A meta-analysis of impacts of alien vs. native plants on pollinator visitation and reproductive success of co-flowering native plants. Ecol. Lett. 12, 716-728.

Ntzoufras, I., 2011. Bayesian Modeling using WinBUGS. John Wiley \& Sons, New Jersey.

Ollerton, J., Killick, A., Lamborn, E., Watts, S., Whiston, M., 2007. Multiple meanings and modes: on the many ways to be a generalist flower. Taxon 56, 717-728.

Ollerton, J., Watts, S., Connerty, S., Lock, J., Parker, L., Wilson, I., Schueller, S.K. Nattero, J., Cocucci, A.A., Izhaki, I., 2012. Pollination ecology of the invasive tree tobacco Nicotiana glauca: comparisons across native and non-native ranges. J. Pollination Ecol. 9, 85-95.

Ollerton, J., Winfree, R., Tarrant, S., 2011. How many flowering plants are pollinated by animals? Oikos $120,321-326$.

Ornelas, J., Ordano, M., De-Nova, A., Quintero, M., Garland, T., 2007. Phylogenetic analysis of interspecific variation in nectar of hummingbird-visited plants. J. Evol. Biol. 20, 1904-1917.
Paradis, E., Claude, J., Strimmer, K., 2004. APE: analyses of phylogenetics and evolution in R language. Bioinformatics 20, 289-290.

Pimentel, D., Lach, L., Zuniga, R., Morrison, D., 2000. Environmental and economic costs of nonindigenous species in the United States. Bioscience 50, 53-65.

Pinheiro, J., Bates, D., DebRoy, S., Sarkar, D., Team, T.R.D.C., 2013. nlme: Linear and Nonlinear Mixed Effects Models. R package version 3, pp. 1-108.

Primack, R.B., Miller-Rushing, A.J., 2009. The role of botanical gardens in climate change research. New Phytol. 182, 303-313.

Core Team, R., 2012. A Language and Environment for Statistical Computing. R Foundation for Statistical Computing, Vienna, Austria.

Richardson, D.M., Allsopp, N., D’Antonio, C.M., Milton, S.J., Rejmanek, M., 2000. Plant invasions - the role of mutualisms. Biol. Rev. 75, 65-93.

Rodger, J.G., van Kleunen, M., Johnson, S.D., 2010. Does specialized pollination impede plant invasions? Int. J. Plant Sci. 171, 382-391.

Rodger, J.G., van Kleunen, M., Johnson, S.D., 2013. Pollinators, mates and Allee effects: the importance of self-pollination for fecundity in an invasive lily. Funct. Ecol. 27, 1023-1033.

Schemske, D.W., Bradshaw, H., 1999. Pollinator preference and the evolution o floral traits in monkeyflowers (Mimulus). Proc. Natl. Acad. Sci. U.S.A. 96, 11910 11915.

Schielzeth, H., 2010. Simple means to improve the interpretability of regression coefficients. Methods Ecol. Evol. 1, 103-113.

Tutin, T., Heywood, V., Burges, N., Valentine, D., Walters, S., Webb, D., 1980. Flora Europaea. Cambridge University Press, Cambridge (1964-1980).

van Kleunen, M., Dawson, W., Bossdorf, O., Fischer, M., 2014. The more the merrier: multi-species experiments in ecology. Basic Appl. Ecol. 15, 1-9.

van Kleunen, M., Dawson, W., Schlaepfer, D., Jeschke, J.M., Fischer, M., 2010. Are invaders different? A conceptual framework of comparative approaches for assessing determinants of invasiveness. Ecol. Lett. 13, 947-958.

van Kleunen, M., Johnson, S.D., Fischer, M., 2007a. Predicting naturalization of southern African Iridaceae in other regions. J. Appl. Ecol. 44, 594-603.

van Kleunen, M., Manning, J.C., Pasqualetto, V., Johnson, S.D., 2008. Phylogenetically independent associations between autonomous self-fertilization and plant invasiveness. Am. Nat. 171, 195-201.

van Kleunen, M., Nanni, I., Donaldson, J.S., Manning, J.C., 2007b. The role of beetle marks and flower colour on visitation by monkey beetles (Hopliini) in the greater cape floral region, South Africa. Ann. Bot. 100, 1483-1489.

Vázquez, D.P., Morris, W.F., Jordano, P., 2005. Interaction frequency as a surrogate or the total effect of animal mutualists on plants. Ecol. Lett. 8, 1088 1094.

Vilà, M., Bartomeus, I., Dietzsch, A.C., Petanidou, T., Steffan-Dewenter, I., Stout, J.C. Tscheulin, T., 2009. Invasive plant integration into native plant-pollinator networks across Europe. Proc. Roy. Soc. Lond. Ser. B: Biol. Sci. 276, $3887-$ 3893.

Vilà, M., Espinar, J.L., Hejda, M., Hulme, P.E., Jarošík, V., Maron, J.L., Pergl, J. Schaffner, U., Sun, Y., Pyšek, P., 2011. Ecological impacts of invasive alien plants: a meta-analysis of their effects on species, communities and ecosystems. Ecol. Lett. 14, 702-708.

Vitousek, P.M., D’Antonio, C.M., Loope, L.L, Rejmanek, M., Westbrooks, R., 1997. Introduced species: a significant component of human-caused global change. N.Z. J. Ecol. 21, 1-16.

Webb, C.O., Donoghue, M.J., 2005. Phylomatic: tree assembly for applied phylogenetics. Mol. Ecol. Notes 5, 181-183.

Wikström, N., Savolainen, V., Chase, M.W., 2001. Evolution of the angiosperms: calibrating the family tree. Proc. Roy. Soc. Lond. Ser. B: Biol. Sci. 268, $2211-$ 2220.

Zuur, A., Ieno, E., Walker, N., Saveliev, A., Smith, G., 2009. Mixed Effects Models and Extensions in Ecology with R. Springer, New York. 